\title{
Etat des lieux de la lutte contre le financement du terrorisme : entre critiques et recommandations
}

State of Affairs in the Fight against Terrorism Financing: Between Criticism and Recommendation

Balances de la lucha contra la financiación del terrorismo: entre críticas y recomendaciones

\section{Anthony Amicelle}

\section{(2) OpenEdition}

\section{Journals}

Édition électronique

URL : http://journals.openedition.org/conflits/16773

DOI : $10.4000 /$ conflits. 16773

ISSN : $1777-5345$

Éditeur :

CCLS - Centre d'études sur les conflits lilberté et sécurité, L'Harmattan

\section{Édition imprimée}

Date de publication : 30 octobre 2008

Pagination : 169-176

ISBN : 978-2-296-06624-3

ISSN : $1157-996 X$

Référence électronique

Anthony Amicelle, «Etat des lieux de la lutte contre le financement du terrorisme : entre critiques et recommandations », Cultures \& Conflits [En ligne], 71 | automne 2008, mis en ligne le 05 février 2009, consulté le 30 mars 2021. URL : http://journals.openedition.org/conflits/16773 ; DOI : https://doi.org/ $10.4000 /$ conflits. 16773 


\title{
Etat des lieux de la lutte contre le financement du terrorisme : entre critiques et recommandations
}

\author{
Anthony AMICELLE
}

Anthony Amicelle, boursier DGA, est doctorant en sciences politiques (relations internationales) à l'IEP de Paris, rattaché au CERI. Ses recherches portent sur la politique européenne en matière de lutte contre le financement du terrorisme et le blanchiment d'argent, sur les enjeux et usages de la surveillance financière et sur les études de sécurité.

Biersteker T.J., Eckert S.E. (eds.), Countering the Financing of Terrorism, New York, Routledge, 2007, 344 p.

Warde I., Propagande impériale et guerre financière contre le terrorisme, Marseille / Paris, Agone / Le Monde diplomatique, $2007,320 \mathrm{p}$.

$\mathrm{O}$ bjet atypique de par l'action publique transversale qu'elle mobilise, la facette financière de l'antiterrorisme a constitué la première réponse de l'administration américaine aux attentats de New York et Washington en 2001. En promulguant le décret présidentiel 13224 le 24 septembre 2001, Georges W. Bush a rendu publique la liste de vingt-sept individus et organisations présumés « terroristes » et a - entre autres choses - ordonné le gel de leurs avoirs financiers. Le front financier a ainsi marqué les prémices de la « guerre contre le terrorisme ». Deux objectifs traversent cet aspect de la stratégie antiterroriste : pister l'argent afin de suivre les « terroristes » à la trace et geler les fonds afin de perturber leurs activités.

Objet atypique, la lutte contre le financement du terrorisme se situe en effet à l'intersection des univers policier (et du renseignement), judiciaire, diplomatique et financier ${ }^{1}$. Cette politique a comme particularité de s'appuyer sur la participa-

1. Favarel-Garrigues G., Godefroy T., Lascoumes P., Les Banques, sentinelles de l'anti-blanchiment: l'invention d'une spécialité professionnelle dans le secteur financier,Paris, CERI, 2006. 
tion d'acteurs privés, notamment du secteur bancaire, mais pas seulement ${ }^{2}$. Instituée dans le cadre plus ancien de la lutte contre le blanchiment de capitaux, cette coopération pour le moins inédite entre professionnels de la sécurité et entités privées joue un rôle décisif dans la chasse aux « finances terroristes », devenue priorité internationale. Outre la transposition de pratiques progressivement institutionnalisées, l'action menée contre le financement du terrorisme emprunte également à l'anti-blanchiment son postulat de départ faisant de l'argent le «nerf de la guerre ». Ce postulat n'a d'ailleurs pas été exempt de critiques dans la littérature scientifique qui s'est largement penchée sur la pertinence de cette focalisation sur les « produits du crime » depuis la fin des années $1980^{3}$. Au-delà de son efficacité, de nombreux travaux ont également étudié les implications du "régime » antiblanchiment, ses ambivalences, la diffusion de normes internationales et leurs réappropriations nationales, ainsi que la question du retrait de l'Etat vis-à-vis de la sphère financière ${ }^{4}$. Toujours est-il qu'au lendemain des attentats de 2001 , les discours officiels ont affiché l'ambition d'assécher les flux financiers en direction des « organisations terroristes » dans le but d'anéantir ou, en tous les cas, de réduire leur capacité de nuisance. "J'ai toujours pensé que le meilleur moyen de stopper le terrorisme est de les frapper [les terroristes] là où cela fait le plus mal, à leurs portefeuilles ». Cette citation résume assez bien la position des tenants de cette facette de l'antiterrorisme au sortir de l'année 2001. Elle démontre aussi la persistance de cette vision à l'égard de la stratégie financière puisqu'elle a été prononcée en avril dernier par le sénateur Chuck Grassley - particulièrement impliqué dans la législation américaine sur ce sujet - au cours d'une audition publique consacrée à l'état des lieux de la lutte contre le financement du terrorisme ${ }^{5}$.

2. Par exemple, la Directive «2005/60/CE du Parlement européen et du Conseil relative à la prévention de l'utilisation du système financier aux fins de blanchiment de capitaux et de financement $d u$ terrorisme » concerne : les établissement financiers et de crédit, les commissaires au compte, experts-comptables externes, conseillers fiscaux, notaires et autres membres de professions juridiques indépendantes, prestataires de services aux sociétés et fiducies, agents immobiliers et, dans certains cas, d'autres personnes physiques ou morales négociant des biens ainsi que les casinos.

3. Voir par exemple : Levi M., "Criminal Asset-Stripping: Confiscating the Proceeds of Crime in England and Wales”, in Edwards A., Gill P. (eds.), Transnational Organised Crime: Perspective on global security, London / New York, Routledge, 2003, pp. 213-225 ; Naylor R.T., Wages of Crime: Black Markets, Illegal Finance and the Underworld Economy, Ithaca / London, Cornell University Press, 2002 ; Van Duyne P.C., "Greasing the Organisation of Crime-Markets in Europe”, in Van Duyne P.C., Von Lampe K., Newell J.L. (eds.), Criminal Finances and Organising Crime in Europe, Nijmegen, Wolf Legal Publishers, 2003, pp. 1-17.

4. Voir par exemple : Andreas P., Friman R.H. (eds.), The Illicit Global Economy and State Power, Lanham, Rowman \& Littlefield, 1999; Beare M.E. (ed.), Critical Reflections on Transnational Organized Crime, Money Laundering and Corruption, Toronto, University of Toronto Press, 2003 ; Favarel-Garrigues G., « "Crime organisé" et lutte anti-blanchiment », in Laroche J. (dir.), Mondialisation et gouvernance mondiale, Paris, PUF, 2003, pp. 161-174 ; Godefroy T., Lascoumes P., Le Capitalisme clandestin : l'illusoire régulation des places offshore, Paris, La Découverte, 2004 ; Schepticky J., "Policing the virtual launderette: Money laundering and global governance", in Shepticky J. (ed.), Issues in Transnational Policing, London, Routledge, pp. 154-160.

5. United States Senate, Committee on Finance, Prepared Statement of Senator Chuck Grassley: "Anti-Terrorism Financing: Progress Made and the Challenges Ahead", $1^{\text {er }}$ avril, 2008, p. 2. 
D'état des lieux, il en est justement question dans deux ouvrages publiés au cours de l'année écoulée et qui se proposent d'analyser les effets des mesures censées s'attaquer au terrorisme par le biais de son financement : Propagande impériale et guerre financière contre le terrorisme d'Ibrahim Warde et Countering the Financing of Terrorism, dirigé par Thomas Biersteker et Sue Eckert. S'ils se différencient sur le ton et la structure, ces deux livres participent explicitement d'un même effort de mise en perspective critique des initiatives prises à l'encontre des « finances terroristes ». La comparaison est d'autant plus intéressante qu'ils s'originent tous deux dans une remise en cause des exagérations et autres approximations qui n'ont cessé d'émailler les discours

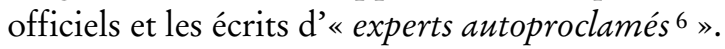

Le premier ouvrage est la traduction en langue française du livre d'Ibrahim Warde intitule The Price of Fear: The Truth Behind the Financial War on Terror sorti également en $2007^{7}$. Professeur associé à la Fletcher School of Law and Diplomacy (Massachussets), Warde nous gratifie ici d'une charge acerbe contre ce qu'il appelle «les guerriers de la finance » en se focalisant sur les Etats-Unis. Discutant point par point les «préjugés initiaux » qui ont forgé la lutte contre le financement du terrorisme, la teneur du propos s'avère particulièrement tranchée :

"Cette politique n'a pas ouvert la moindre brèche dans le financement du terrorisme. En revanche, elle a remodelé le système financier planétaire et produit des effets importants mais bien souvent non désirés dans les domaines politique et économique ${ }^{8}$. »

Construit en cinq parties, l'argumentaire passe en revue les fausses convictions qui ont accompagné la mise en priorité de l'enjeu « financement du terrorisme » et qui continuent en partie à imprégner cette dimension de l'antiterrorisme. L'auteur revient sur la problématique de la mesure du phénomène et des chiffres tels que le mythe des 300 millions de dollars de Ben Laden, les évaluations hasardeuses d'une «économie de la terreur ", ou encore l'idée même de l'argent comme oxygène du terrorisme. Il pointe la faible consistance méthodologique d'estimations récurrentes, citant en particulier Loretta Napoleoni qui évalue cette «économie de la terreur » à quelques 1500 milliards de dollars et Richard Labevière qui a, quant à lui, apprécié la fortune de Ben Laden à près de 3 milliards de dollars ${ }^{9}$. Faisant régulièrement référence aux conclusions de la

6 . Thomas Biersteker et Sue Eckert utilisent l'expression «self-proclaimed experts» dans la préface de leur ouvrage, sans toutefois spécifier explicitement à qui elle se rapporte.

7. Warde I., The Price of Fear: The Truth behind the Financial War on Terror, London, I. B. Tauris \& Co. Ltd, 2007.

8. Warde I., Propagande impériale et guerre financière contre le terrorisme, Marseille / Paris, Agone / Le Monde Diplomatique, 2007, p. 25.

9. Voir :Napoleoni L., "Money and Terrorism”, Strategic Insights, vol.3, n 4, 2004 ; Labevière R., Dollars for Terror: The United States and Islam, New York, Algora Publishing, 2000, 
Commission d'enquête sur le 11 septembre 2001 10, Ibrahim Warde s'emploie à dissiper les considérations abusives quant aux liens entre activités terroristes et certains «usual suspects». Cette dernière expression recouvre les développements sceptiques de l'auteur sur les hypothèses établissant une connexion entre criminalité organisée et terrorisme, entre diamants et finances terroristes ou bien stigmatisant les systèmes des « Hawalas 11 » et des organisations caritatives. Cette démonstration autour des « suspects habituels »-plus ou moins convaincante selon le thème abordé - permet au chercheur de mettre en exergue quelques effets pervers découlant de renseignements limités et de stigmatisations précipitées. Il insiste notamment sur la fermeture abrupte de l'entreprise somalienne de transfert de fonds Al-Barakaat, accusée par les Etats-Unis d'avoir financé AlQaïda à hauteur de plusieurs dizaines de millions de dollars ${ }^{12}$. Les renseignements ayant entraîné cette fermeture extrêmement médiatisée ainsi que le gel des fonds et des transactions des agences d'Al-Barakaat n'ont pas permis d'aboutir à une condamnation formelle de l'entreprise. Manquant pour certaines de crédibilité, ces informations se sont donc révélées insuffisantes et la majorité des fonds gelés aux Etats-Unis a finalement été restituée. Cette opération stérile contre le terrorisme n'a pourtant pas été sans conséquences dommageables pour la population somalienne étant donné le rôle économique des envois de fonds de la diaspora vers ce pays et la place de premier ordre qu'occupait Al-Barakaat.

Le cœur de l'argumentation vient questionner à juste titre la pertinence de l'usage de l'« arsenal anti-blanchiment » pour combattre le financement du terrorisme. Warde s'attarde sur les évolutions de la politique contre le blanchiment d'argent et met ainsi en lumière les confusions et dysfonctionnements issus du glissement de priorité en 2001. En plus d'un recyclage des dispositions, ce sont aussi les acteurs et institutions engagés précédemment dans la lutte contre l' « argent sale » du trafic de drogues et de la criminalité organisée qui ont dû se focaliser assez soudainement sur le « terrorisme ». Il rappelle les différences existantes entre les deux phénomènes et les réticences des experts du Groupe d'action financière (GAFI), début 2001, sur la pertinence d'user des instruments contre le blanchiment pour lutter contre le financement du terrorisme ${ }^{13}$. Et de manière intéressante, l'auteur décrit le «virage à $180^{\circ}$ »

p. 117 (traduction française : Les Dollars de la terreur. Les Etats-Unis et les islamistes, Paris, Grasset, 1999).

10. Roth J., Greenburg D., Wille S., National Commission on Terrorist Attacks upon the United States, Monograph on Terrorist Financing, 2004, disponible sur : http://www.9-11commission.gov/staff_statements/911_TerrFin_Monograph.pdf

11. Il s'agit de systèmes informels de transfert d'argent. Warde souligne que "si ce sytème est effectivement très utilisé par certaines communautés, en particulier dans les régions du monde où le service bancaire est trop coûteux ou trop rudimentaire, voire inexistant, il n'est pas nécessairement clandestin ".

12. Statement by Treasury Secretary Paul O'Neill, 7 novembre 2001. Disponible sur: http://www.treas.gov/press/releases/po770.htm

13. Warde I., Propagande impériale et guerre financière contre le terrorisme, op. cit., p. 106. Le Groupe d'action financière (GAFI) est un organisme intergouvernemental visant à dévelop- 
qu'a représenté le 11 septembre 2001 dans la politique et les comportements du gouvernement fédéral vis-à-vis de la législation contre le blanchiment d'argent. L'administration Bush a subitement échangé sa position critique contre un soutien indéfectible à la réglementation anti-blanchiment, désormais incorporée dans l'action globale contre le terrorisme.

Ce retournement conjoncturel est également relaté dans l'ouvrage collectif coordonnée par Thomas Biersteker et Sue Eckert ${ }^{14}$. Principaux instigateurs du Targeted Terrorist Finances Project développé au Watson Institute for International Studies (Brown University), ces deux internationalistes ont donc dirigé ce livre avec pour ambition d'allier évaluation critique et recommandations. Distribuées en trois parties bien distinctes ${ }^{15}$, les onze contributions prises dans leur ensemble se proposent d'interroger les cinq hypothèses qui soustendraient la réponse réglementaire américaine et internationale contre le financement du terrorisme. Les différents chapitres tentent donc d'objectiver ces suppositions enchâssées dans l'approche financière de l'antiterrorisme :

- l'idée que tous les groupes ou organisations engagés dans des actes de terrorisme sont essentiellement les mêmes;

- l'idée que les institutions financières du secteur formel sont ou peuvent être les principales sources de transferts de fonds terroristes;

- l'idée que la réglementation des institutions financières formelles peut être étendue aux opérations des réseaux financiers informels et aux autres sources potentielles de transferts financiers;

- l'idée que le système « Hawala » et des systèmes informels similaires de transfert de valeurs opérant dans d'autres parties du monde jouent un rôle important dans le financement du terrorisme ;

- l'idée qu'il y a un lien (nexus) émergent entre crime organisé et terrorisme.

Globalement, les lignes directrices qui en ressortent appellent à une approche moins homogénéisante et donc plus différenciée qui puisse tenir compte des évolutions organisationnelles et des multiples distinctions entre groupes ciblés. La critique sous-jacente envers une réponse réglementaire partiel-

per et promouvoir des politiques nationales et internationales afin de lutter contre le blanchiment de capitaux et le financement du terrorisme. Le mandat du GAFI s'est officiellement élargi au financement du terrorisme fin octobre 2001.

14. Biersteker T.J., Eckert S.E. (eds.), Countering the Financing of Terrorism, New York, Routledge, 2007, pp. 213-214.

15. Part I: The social organization of terrorism; Part II: The financial organization of terrorism: the raising and movement of funds; Part III: Responses to the terrorist financing challenge. 
lement aveugle aux particularités vaut aussi à l'égard des systèmes et des institutions financières qu'elle vise à réguler. De ce point de vue, la contribution de Nikos Passas et Samuel Munzele Maimbo sur les systèmes informels de transfert de fonds est extrêmement intéressante. S’appuyant sur leurs précédents travaux, les deux auteurs donnent à voir les implications d'une vision ethnocentrée sur ces pratiques informelles tout en soulignant les enjeux économiques et les réels défis liés aux tentatives de réglementation de ces systèmes. A travers une démarche comparative entre Al-Qaïda et l'organisation palestienne Hamas, Jeroen Gunning apporte quant à lui des éclaircissements sur la nature du lien entre le financement de ces entités et des organisations caritatives musulmanes européennes. Au-delà d'une critique nuancée des « narratifs populaires » sur cette question, Gunning se concentre sur les singularités des deux entités qui permettent en partie de comprendre les types de relations particulières qu'elles entretiennent avec des associations à but non lucratif. Devant les conséquences potentielles d'une fermeture d'organisations caritatives, il interroge la portée de l'incrimination « financement du terrorisme » et l'impact que peuvent avoir les différences de traitement à l'égard d'associations suspectes selon les pays concernés ${ }^{16}$.

Ces études sur des points spécifiques alternent avec des chapitres plus généraux, dont certains retracent les initiatives américaines et internationales en analysant leurs effets concrets ainsi que les enjeux qui s'y rapportent. Sue Eckert brosse par exemple un portrait très instructif et détaillé de l'évolution réglementaire des Etats-Unis. Elle montre comment les attentats ont entraîné le déploiement de nouvelles mesures contre les fonds «terroristes » mais aussi, et surtout, une transposition discutable de méthodes et de techniques préexistantes. A la manière d'Ibrahim Warde, Eckert souligne les tensions qui se sont faites jour autour du rapport entre coût et bénéfice de l'antiblanchiment avant de faire place à une inflation législative dans le sillage des événements du 11 septembre 2001. Elle laisse également entrevoir la transition entre une première phase focalisée sur les désignations publiques et le gel des avoirs et une deuxième, davantage axée sur le partage et l'analyse d'informations financières en tant qu'outils de compréhension des réseaux terroristes 17. Cela lui permet de spécifier le rôle central des acteurs privés - qui n’est pas sans poser certains problèmes - et l'évolution de leurs relations avec les professionnels de la sécurité tout en questionnant la capacité de ces derniers à exploiter les volumes d'informations reçues.

Bien des points évoqués ci-dessus se retrouvent sous une forme ou sous une autre chez Warde. De prime abord, la mise en perspective de ces deux ouvrages laisse apparaître un certain nombre de convergences qui viennent pointer les limites et les «dommages collatéraux » imputables à la stratégie menée contre le

16. Gunning se penche sur les différences qui existent entre les gouvernements britanniques et néerlandais dans la façon de superviser et contrôler les organisations caritatives suspectes.

17. Biersteker T.J., Eckert S.E. (eds.), Countering the Financing of Terrorism, op. cit., p. 216. 
financement du terrorisme. En dehors de leur évaluation critique respective, ces textes montrent bien le rôle de catalyseur de l'enjeu «financement du terrorisme », au nom duquel le régime anti-blanchiment a été étendu et transformé. A l'image des dispositions du USA Patriot Act, des mesures qui avaient fait l'objet de blocages et de vives résistances de la part des institutions financières ou des défenseurs de la vie privée ont été promptement adoptées au Congrès sans trop de débat. De nombreux processus législatifs ont donc abouti au nom de la " guerre financière contre le terrorisme "; une des conclusions de Biersteker et Eckert sur les externalités positives de cette lutte l'illustre parfaitement. Parmi les bénéfices issus des initiatives engagées, ils insistent ainsi sur l'utilité de ces larges mesures pour appuyer les efforts réglementaires contre l'évasion fiscale, la corruption ou encore contre certains Etats tels que l'Iran ou la Corée du Nord, soumis à des sanctions multilatérales ${ }^{18}$. A peine après avoir évoqué ce qu'ils assimilent à des "positive spillovers 19 ", ces auteurs tempèrent leur propos en rejoignant les développements d'Ibrahim Warde sur les inquiétudes en termes de droits fondamentaux inhérentes aux procédures de désignation et de retrait des «listes terroristes». Ils détaillent les efforts limités de réformes qui ont été consenties au niveau des Nations unies et se font l'écho des critiques persistantes sur les droits de recours des individus et entités listés.

Toutefois, là où les conclusions de Warde s'avèrent résolument critiques si ce n'est exclusivement critiques, celles de l'ouvrage collectif se veulent plus nuancées tout en partageant en grande partie le scepticisme informé de leur collègue. Celui-ci ne nie pas l'" outil formidable de renseignements et de travail de police $20 »$ que peut représenter le financement mais il se borne à une condamnation en bloc de l'approche mise en œuvre sans développer outre mesure. Le second livre explicite davantage les fonctions attendues (en théorie) des contrôles financiers, au titre desquelles : une fonction dissuasive, analytique (surtout investigations post-attentats) et préventive sans oublier une utilité politique (dimension symbolique). S'ils ont tendance à reconnaitre l'impact dissuasif et surtout l'intérêt des données financières lors des investigations après des opérations violentes, les contributeurs sont beaucoup plus dubitatifs sur la capacité de détection et de prévention de futurs actes terroristes. Les deux ouvrages dressent dès lors une vision plus modeste de la lutte contre le financement du terrorisme, de ce qu'il faut en attendre, et prennent leur distance avec les potentialités d'anticipation souvent prêtées à la surveillance financière.

Même s'ils ont été publiés à la même période, certaines contributions de l'effort collectif retranscrivent mieux les évolutions récentes de la lutte contre le financement du terrorisme que Warde, dont les arguments s'attardent parfois sur des points qui paraissent quelque peu datés. Mais ce qu’il peut gagner en préci-

18. Ibid., p. 251 et p. 254.

19. Externalités positives de la lutte contre le financement du terrorisme.

20. Warde I., op. cit., p. 253. 
sion, l'ouvrage collectif tend quelquefois à le perdre en homogénéité voire en cohérence. En effet, il est permis de douter de l'existence d'une ligne de pensée commune entre les chapitres de Jeroen Gunning, de Michael Levi et celui de Phil Williams par exemple. Les deux premiers auteurs se méfient des notions «parapluies » que sont le «terrorisme » ou encore le «crime organisé » dont la dimension englobante peut entraver la compréhension de faits sociaux extrêmement différents et interférer avec les efforts des stratégies de contrôle. Malgré une prudence affichée sur les réalités d'un lien (nexus) entre crime organisé et terrorisme, Phil Williams adopte à l'inverse une approche réifiant cette terminologie puisqu'il en vient à parler d'«essence » du terrorisme et du crime organisé 21 . Loin de toute analyse critique des notions utilisées mais toujours sous couvert de prudence, il n'hésite pas affirmer qu'il est hautement probable que dans le futur les connexions entre organisations terroristes et criminelles deviennent de plus en plus fréquentes et importantes ${ }^{22}$. Dans la même veine téléologique, l'auteur n'hésite pas non plus à lancer l'hypothèse selon laquelle la plus sérieuse menace à long terme pourrait bien être la transformation de criminels en terroristes ${ }^{23}$, et ce à partir d'une généralisation contestable basée sur les attentats de Madrid en 2004. Il est donc aisé ici de relever une relative discordance entre certaines contributions, mais cette précision est moins une critique recevable qu'un constat de la diversité des approches composant ce livre.

Toujours est-il que ces deux ouvrages nous offrent un panorama très éclairant des enjeux et des pratiques que recouvre la facette financière du contre-terrorisme. Par le biais d'une contextualisation diachronique, ils montrent que ces politiques contre les «finances terroristes» ne sont pas nées ex nibilo, mais s'inspirent à la fois des sanctions économiques internationales et des méthodes anti-blanchiment. Leurs analyses les conduisent à souligner les tensions et les effets parfois contre-productifs résultant de cette stratégie mise en œuvre depuis 2001 et qui peine à être réévaluée et adaptée. Bien que cela n'ait été leur objectif, on peut tout de même regretter que ces travaux captivants n'aient pas tenté de tirer des conclusions plus générales sur les relations entre acteurs publics et privés ainsi que sur le modèle de surveillance des flux financiers qui s'en dégage. Objet atypique, la lutte contre le financement du terrorisme peut en effet constituer un de ces «objets empiriques à propos desquels on peut poser réellement des problèmes très généraux ${ }^{24}$ ».

21. De ce point de vue, la contribution de Phil Williams n'est pas sans rappeler ces travaux antérieurs sur le "crime organisé transnational»; voir par exemple Williams P., BaudinO’Hayon G., "Global governance, transnational organised crime and money laundering”, in Held D., Mc Grew A. (eds.), Governing Globalization, Cambridge, Polity Press, 2002, pp. 127-143; Williams P., "Transnational criminal organzations and international security", Survival, vol.36, $\mathrm{n}^{\circ} 1$, printemps 1994, pp. 96-113.

22 . Biersteker T.J., Eckert S.E. (eds.), op. cit., p. 136.

23. Ibid., p. 145.

24 . «Un des secrets du métier de sociologue consiste à savoir trouver les objets empiriques à propos desquels on peut poser réellement des problèmes très généraux ", Bourdieu P., Questions de sociologie, Paris, Les Editions de Minuit, 1984/2002, p. 51. 